\title{
Evaluation of the Storage Life of Gyroscope Based on Accelerated Life Test
}

\author{
Chen Huang ${ }^{1, a, ~}{ }^{*}$, Xihui $\mathrm{Mu}^{2, \mathrm{~b}}$, Yueting $\mathrm{Niu}^{2, \mathrm{c}}$ \\ ${ }^{1}$ Mechanical Engineering College, Shijiazhuang 050000, China \\ ${ }^{2}$ New Technology Research Institute, Shijiazhuang 050000, China \\ a13273172420@163.com, b15637456237@163.com, c3795463587@qq.com
}

Keywords: gyroscope, accelerated life test, life assessment.

\begin{abstract}
In this paper, the life evaluation of gyroscope components of information-based ammunition control system is carried out by using accelerated life test. The present research situation of accelerated life test technology is discussed, and the basic principle, shortcomings, the general process of evaluating the life of the gyroscope components of the control system by the acceleration test and some problems needing attention in the life assessment process are put forward.
\end{abstract}

\section{Introduction}

Information ammunition refers to the new ammunition that can use information technology from a complex background to detect, identify, track the target, and guide, control the projectile flight, hit and damage targets through precision guidance system with the missile as a carrier platform. China's information ammunition reserves a lot, and plays an important role with its high value. In recent years, a large number of information ammunition have reached a given storage period, the demand for life assessment is increasingly urgent, and need for in-depth study. The control system in information ammunition is its "brain and central nervous system", with a large number of high-tech and materials, its complexity is far higher than ordinary ammunition, so its failure mode and life law is difficult to grasp, and we need carry out its life assessment ${ }^{[1]}$.

The gyroscope is a high-precision, automatic north-looking navigation instrument whose accuracy and reliability are directly related to the level and performance of the control system. Traditional evaluation of the life of the electromechanical products such as gyroscopes is often based on the monitoring of the natural environment test life assessment method, but using this method to assess the gyroscope evaluation cycle is so long that can not apply to modern mechanical and electrical products rapid replacement speed. Therefore, the current method to implement the control system gyroscope component life assessment is mainly based on the accelerated test life assessment at home and abroad.

\section{Life status assessment based on accelerated life test}

\subsection{Basic concepts related to life assessment}

In the ammunition system, a very critical technical indicator is the shelf life, so the evaluation of the ammunition storage life is a vital task. Ammunition storage life assessment technology in the world's attention has been rapid developed ${ }^{[2]}$.

The evaluation technique of storage life mainly includes two parts: storage test monitoring technology and accelerated life test evaluation technology in natural environment. Natural environment storage test monitoring technology, that is, putting the relevant samples in a typical natural environment and monitoring the performance of their changes after long-term storage, according to certain criteria to determine its storage life. Natural environment storage test monitoring technology focuses on its environmental adaptability evaluation, environmental failure mechanism analysis, environmental protection measures, and then combined with life test data analysis, 
predicting its service life at last. Although the test cycle of the natural environment storage test is longer, the data are more realistic and reliable ${ }^{[3,4]}$.

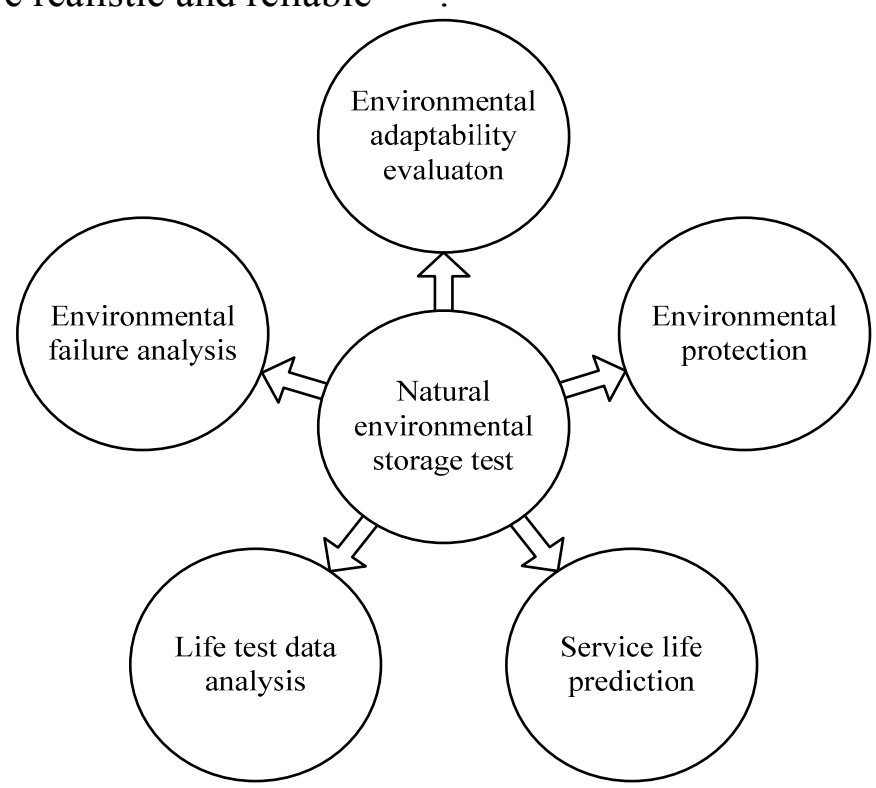

Figure. 1 Natural environmental storage test

The gyroscope discussed in this paper is the key part of some intelligent ammunition control system, and its accuracy and reliability are directly related to the level and efficiency of carrier work. Material quality, parts processing accuracy and other factors will affect its life, domestic and foreign evaluation of the life of the gyroscope is mainly using real-time 1: 1 continuous life test, but this method requires more sub-number, and will bring to great financial, material and time wasted. Beijing University of Aeronautics and Astronautics, astronautical institutions and other units of fiber optic gyroscope reliability research mainly study from the performance degradation and fault tree and other aspects ${ }^{[5]}$. Shanghai Jiao Tong University has carried on the life test to the relatively simple dynamic gyroscope, and uses the parameter performance extrapolation method to preliminarily discuss the life expectancy of the gyroscope ${ }^{[6]}$. In this paper, the use of accelerated life test technology to predict the life of the gyroscope, greatly improving the cost of gyroscope life prediction, has important practical significance ${ }^{[7]}$.

\subsection{Summary of research at home and abroad}

At present, Russia and the United States in the new ammunition storage life assessment technology are the world leaders in the application of new ammunition life prediction and reliability analysis has made many more mature results. It has been widely used in new ammunition rubber, propellant, propellant, combustible cartridge and electronic parts, optical parts, etc., which have used the combination of "storage test of natural environment storage test and accelerated life test evaluation "method, but the technical approach between Russia and the United States is slightly different.

The United States mainly uses natural storage test technology assisted by accelerated life test. Their main experience is to implement the storage reliability plan from the time of the delivery of new ammunition, to monitor the aging trend of the product throughout the storage period; to provide a life prediction technology for accelerated life testing as a new ammunition, using accelerated aging technology 48 months of service life forecast. The United States carried out the CD4011 "real life plan" and "accelerated life plan" ${ }^{[8]}$, through its storage of smart ammunition to take functional monitoring, laboratory testing and flight testing and other related means to assess the combat performance and other conditions for its life assessment as data support. Such as "Dow Anti-Tank Missile" after a comprehensive functional testing, laboratory testing, flight test, the life expectancy changed from the original 5 years to 22 years. By using natural storage monitoring test, the storage environment is real, the test results are credible, not subjected to the size of the test piece size; but the test cycle is longer, generally a few years or even ten years.

In contrast, in the information ammunition detection technology, monitoring means, China is still relatively backward, its roots lie that the basic work is weak, the standard is not perfect. The United 
States monitoring means is advanced, as early as in the eighties of last century to carry out the "remote monitoring plan", relying on micro-sensors, satellites and other remote monitoring of missile temperature, humidity, vibration, shock, once the monitoring data beyond the allowable value, data is uploaded to the data center alarm. At present, China can only carry out regular inspection and maintenance of new ammunition, some new ammunition does not have the means of detection, some of the existing means of detection is self-contained with single function, and China did not establish a corresponding monitoring database.

Russia mainly uses accelerated life test technology to evaluate the new ammunition storage life, supplemented by natural storage test technology. Its outstanding feature is that accelerated life test level is higher and can be full-level. The key to accelerating the storage life test technique is to accelerate the establishment of an accelerated storage life model based on the physical model of material aging. There are many types of stress types that affect the aging of the material. In the Russian national standard ГоСТ9.707, the dynamic process of more than 20 stress types is described [9], which need to be input to the accelerated test software before the accelerated life test. Accelerated storage life test software is based on the mathematical model of the testing state. To design the accelerated storage life test program, the following data must be input to the software: A,Storage environment condition statistics (temperature, humidity, etc.); B , Storage sites; C, sample material list, including a detailed list of each material and its energy characteristics; D, the number of part assembly, status and characteristics of the sample, and its storage failure rate and storage life.

Accelerated storage life test program formation process is shown in Figure 2. According to the test scheme, various stress types such as temperature and humidity are controlled by a computer in accordance with a predetermined law. In the accelerated life test of the first product, there are more failures, and analyze the cause of the malfunction, then take measures, and put this measure into the product design specifications or process specifications, over time, to speed up the storage life test results to make it more accurate.

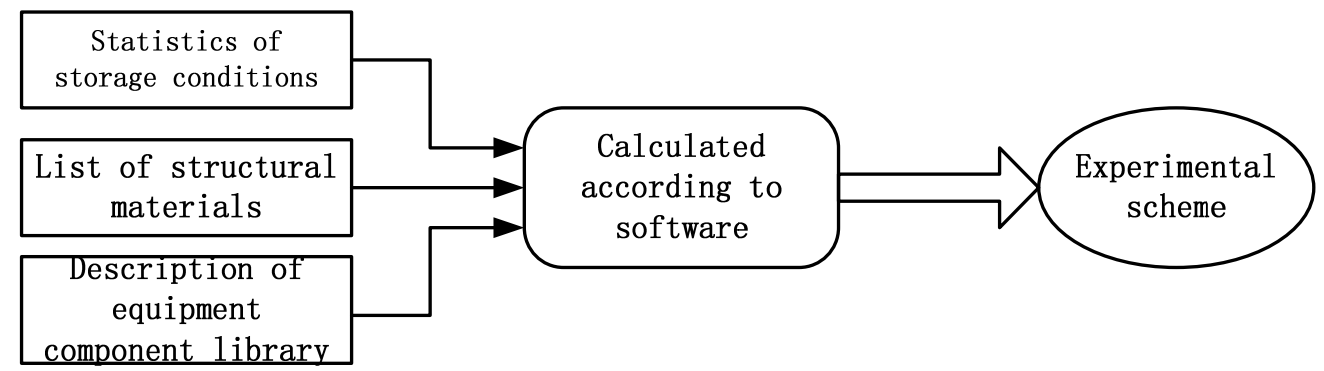

Figure. 2 Russian accelerated storage test program formation process

By accelerating the storage life test, Russia was able to find weaknesses in missiles during the engineering development phase, record its accelerated aging process, and design improvements of the product to derive the inherent shelf life of the product. For example, Russia processed an accelerated life test of a defensive missile for 6 months and make conclusion that its storage life will be10 years.

Russian experts believe that the accelerated life test has a short test cycle characteristics, which can be developed in the product stage of the product shelf life, you can simulate the use of many environmental conditions; and it can improve the design based on accelerated life test results before mass production so as to avoid the waste after the design. But limited to the test technology, there is still a certain gap between the results through the accelerated life test and the actual situation, and it need to be compared with field storage test results.

As the system structure of intelligent ammunition is complicated, the accelerated life test is an experimental category of accelerated life of complex system ${ }^{[10,11]}$. There are multiple failure mechanisms in the complex system of intelligent ammunition. System failure is the result of competing with multiple potential failure mechanisms. At present, the research on the accelerated life test of complex systems under the condition of competition failure has aroused the attention of scholars at home and abroad, and the relevant discussion will still be the focus of research in the future for a period of time ${ }^{[12]}$. 
China actively uses foreign military research results, combining accelerated life test technology and natural storage test technology to study the new ammunition life assessment. In the 1960s, field tests were carried out on tactical missiles and strategic missiles, liquid propellant missiles and solid propellant missiles, various components and materials, and produced significant military and economic benefits such as anti-tank missiles from the early factory given 5 years of life, after the scientific life assessment to determine its reliable storage life of 8 years; a missile storage life of the original 5 years, after life assessment and repair, can directly be extended to 20 year; a missile after life assessment, testing and repair, the storage life extended from 7.5 years to 15 years.

Although our country has gained some experience and theory in the evaluation of the storage life of the missile, these research results are not fully applicable to the new ammunition studied in this project. We can't evaluate reliable storage life only when choosing different methods and conditions of different types of ammunition, and then study its life extension repair technology.

\section{Accelerated Life Test Overview}

As the most commonly used test technology for complex equipment reliability assessment, accelerated test technology is an emerging engineering technology developed to evaluate the reliability of the product and to cover a wide range of products. It began in the 1950s, specializing in research from the $1960 \mathrm{~s}$, after 70 years of technical theory mature and 80 years of further vertical and horizontal development, accelerated test technology has entered a deeper and broader multi-disciplinary cross-cutting new areas since the $1990 \mathrm{~s}$, and to practical engineering transformation of the new stage.

\subsection{Concept definition}

In 1967, the United States Roma Aviation Development Center define the accelerated life test for the first time: Accelerated Life Test (ALT) is a test that using reasonable acceleration model of the product in the accelerated environment to analysis the characteristics of the product relying on reliability theory and engineering practice so as to reach the extrapolated product under the normal working stress ${ }^{[5,13-16]}$. Accelerated life test is mainly suitable for the product that failure mode is the sudden failure mode.

\subsection{Accelerated test type}

The stress type can be divided into three types according to the stress load: the constant stress acceleration test (constant plus test), step plus stress acceleration test (step test), sequential stress acceleration test (sequence test) can be divided as shown in Fig.3. For the above three kinds of accelerated test methods are described as follows:

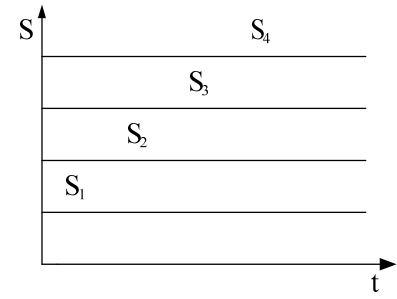

(a)

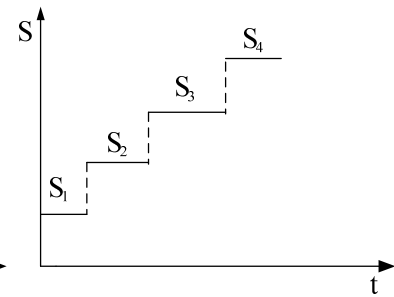

(b)

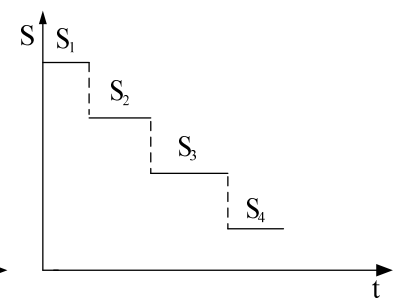

(c)

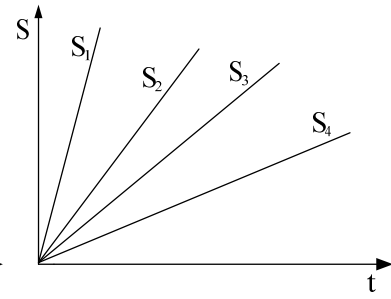

(d)

Figure.3 Accelerated test stress type

(a) constant test; (b) step test; (c) step-down test; (d) sequential test

1) Constant stress acceleration test $[5,17,18]$

Select a set of stress levels above the normal stress level, the test sample according to the stress level will be assigned to different stress level and tested until to cut-off conditions.

2) Step plus stress acceleration test ${ }^{[5,17,18]}$

Select a group of stress levels $\mathrm{S}_{1}, \mathrm{~S}_{2} \ldots \mathrm{S}_{\mathrm{k}}$ higher than the normal stress level $\mathrm{S}_{0}$ (according to a certain size order, can be gradually increased step by step, can also be gradually reduced to step down),put all samples to test at the stress level $\mathrm{S}_{1}$, then change the stress level to $\mathrm{S}_{2}$ until the test 
cut-off condition is reached at stress level $S_{1}$ and repeated until the unexpired specimen reach the cut-off condition at the stress level $\mathrm{S}_{\mathrm{k}}$.

Step plus stress acceleration test can be divided into step test and step down test, domestic and foreign related research results show that ${ }^{[19-22]}$, for long-life, highly reliable products, step down test can shorten the test time, so it's more efficient and obvious. Because the step-down test is to start from the high stress on the product life loss, then reduce the stress level when the failure begins, but the sample has undergone a period of high stress levels, so from the test point of view, the test time will be shorten, so the product failure or life information can be accessed quickly.

Unlike the constant test, the sample in the step test is often subjected to multiple stress levels, which gives it the advantage of quick failure and low number.

3) Progressive stress acceleration test ${ }^{[5,17,18]}$

Select a set of stress levels for the time-increasing function, the general selection of the stress level is proportional to the relationship between the level of stress. Due to rapid changes in the level of stress, the occurrence of sample failure is also fast ${ }^{[5]}$.

The above three kinds of accelerated test in our country have been applied, but the constant application was most widely used, and test and evaluation technology is also the most mature. Step (including step and step-down) test is currently in the research stage, it can improve the efficiency of the experiment, there are a small amount of application. The shortcomings of the sequential test in equipment and data analysis make it difficult to actually apply to engineering ${ }^{[16]}$. For the products with long life and high reliability, in order to further shorten the test time and improve the test efficiency to meet the realistic engineering requirements, the acceleration test can be used in the form of step test, so this paper will focus on the control system gyroscope components step stress accelerated test.

\section{Evaluation of storage life of gyroscope components}

Based on the accelerated life test of the life assessment program of the basic idea is:

\begin{tabular}{|c|c|c|c|}
\hline $\begin{array}{l}\text { accelerate } \\
\text { d stress } \\
\text { method }\end{array}$ & $\begin{array}{l}\text { Accelerated } \\
\text { life testing } \\
\text { model }\end{array}$ & \begin{tabular}{|c} 
Product \\
life \\
distributio \\
$n$
\end{tabular} & $\begin{array}{c}\text { Life } \\
\text { evaluation } \\
\text { method }\end{array}$ \\
\hline
\end{tabular}

Figure. 4 basic assessment of life assessment

(1) To determine the way to accelerate the application of stress.

In general, the application of accelerated stress is mainly mentioned in the above three, namely constant stress method, step stress method and the progressive stress method, we can according to the specific purpose of the sample pre-accelerated test method to determine the way take the stress applied.

(2) To determine the acceleration of the accelerated life test model.

Accelerated model (also known as acceleration equation): the equation used to express the relationship between life characteristics and stress levels. The acceleration model is derived from testing the critical factors of the product under normal stress levels and at one or more levels of accelerated stress. Be very careful when using an accelerated environment in order to identify and correctly identify failures that will occur in normal use and generally do not occur. The five most commonly used acceleration models in the acceleration test are: Alanius model, inverse power law model, single stress Eyring model, generalized Eyring model, temperature-nonthermal model.

Different acceleration models are suitable for different accelerating stresses and need to be used to determine the applicable acceleration model based on the type of stress that affects the shelf life of key components within the control system.

(3) To determine the product life distribution.

In general, it is possible to obtain the lifetime distribution function by combining the natural storage test data of the sample and accelerating the test data. There are three distributions of the 
product's life distribution: exponential distribution, Weibull distribution, logarithmic normal distribution.

(4) To determine the method of life estimation.

For the creep life data or the whole life data processing of the accelerated storage life test, there are maximum likelihood estimation method, linear estimation method and graph estimation method. In the life estimation method of the small sample, the contraction estimation of the fusion prior parameter information has better statistical properties than the maximum likelihood estimation.

\section{Conclusion}

Foreign countries have carried out more research on the evaluation of the life of information ammunition, Europe and the United States and Russia has been able to achieve system-level life assessment,and life prediction and reliability analysis used in weapons and equipment have made many more mature results. At present, China is in the tracking and preliminary research stage in the accelerated life test: it's not long since China begin to carry out accelerated life test research, coupled with foreign technology blockade,so there is still a huge gap compared with developed countries in hardware and software. There is still no practical and feasible experimental design method for the accelerated life test of informatization ammunition. We need to deepen the theory of accelerated life test, which is about the sub-system of the evaluation of the storage life of information ammunition and the acceleration test of the whole bomb system research and improve the accelerated life test method of technical means.

\section{References}

[1] Wang Dongmei, Dai Wenyong, Zhang Yongtao. Research Status and Development Trend of Information Ammunition [J] .Journal of Ordnance, 2010, (32).

[2] Zhang Xiaoli, Chen Xuefeng, Li Bing.Study on Life Prediction of Major Mechanical Equipment [J]. Journal of Mechanical Engineering, 2011, (11).

[3] Zeng Shengkui. Reliability design and analysis [M]. Beijing: National Defense Industry Press, 2013.

[4] Hao Chong, Xu Lutie, Lv Shuai.Application of Accelerated Life Test Technology in Ammunition Storage Reliability Engineering [J]. Equipment Environmental Engineering, 2012, (05): 48-51

[5] Zhang Chunhua. Overview of accelerated life test technology [J]. Journal of Ordnance Industry, 2004, 25 (4): 485-490.

[6] Zhang Sheng-peng, Wang Xiaohong, Li Xiaogang.Design of Accelerated Storage Test Scheme for Electronic Machine [J]. Quality and Reliability, 2011,152 (2): 24-28.

[7] Peng Zhiling, Yang Jinwei, Shao Yiqun.Fan Xiaodong.Life Prediction of Liquid-floating Gyroscope Based on Accelerated Life Test [J]. Journal of North China University (Natural Science Edition), 2014,35 (6): 662-665

[8] Joseph Martinelli Pecht Associates. Effect of Long-Term Storage on Electronic Devices [R]. U.S. Army Armament Reserch, Development and Engineering Center, 1995.11.

[9] Zhang Hong,Hou Xiji. Russia to accelerate storage and accelerated transport test technology overview [J]. Overseas study technical report, 1997, 2: 109-113.

[10] Gao Meng, Wang Jinzhu, He Xueguang. Evaluation of the life of guided ammunition based on reliability statistical analysis. Equipment Environmental Engineering[J].2013, 10 (3): 110-113.

[11] Zhou Jie, Yao Jun, Song Yan. Storage life evaluation method based on piecewise nonlinear Arrhenius [J]. Journal of Beijing University of Aeronautics and Astronautics, 2015, 41 (4): 744-750.

[12] T.A. Mazzuchi C. Bunea. Competing failure modes in accelerated life testing [J]. Journal of Statistical Planning and Inference, 2006, 136: 1608-1620.

[13] Schafter R E Finkelstein J. M. Yurkowsky W. Accelerated testing technology, RADC-TR-67-420 [R]. Rome Air Development Center, 1967. 
[14] Nelson W B. Accelerated testing: statistical models, test plans, and data analyses [M]. New York :: John Wiley \& Sons, Inc., 1990.

[15] ESCOBAR L A MEEKER W Q, LU J C. Accelerated off tests: Modeling and analysis [J]. Technometrics, 1998, 40 (2): 89-99.

[16] Tan Yuanyuan. Equipment storage life machine acceleration test technology research [D]. Changsha: National University of Defense Technology, 2010.

[17] Wang Lingling Mao Shisong. Accelerated life test [M]. Beijing: Science Press, 2000.

[18] Chen Shou, Zhang Chunhua, Wang Yashun, Tan Yuanyuan. Accelerated life test technology and application [M]. Beijing: National Defense Industry Press, 2013.

[19] Wang Yashun, Zhang Chunhua, Chen Zhan. Step-down stress accelerated life test (sequel) Optimization design [J]. Journal of Ordnance, 2007, 28 (6): 686-691.

[20] Zhang Chunhua. Theory and method of step-down stress accelerated life test [D]. Changsha: National University of Defense Technology, 2002.

[21] Zhang Chunhua, Chen Xun, Wen Xisen. Step-down stress accelerated life test (Part II) Statistical analysis [J]. Journal of Ordnance, 2005, 26 (5): 666-669.

[22] Chunhua Zhang, Xun Chen, Yashun Wang. A New Step Stress Accelerated Life Testing Methodology: Step-down-stress. In Proceeding of 2008 European Safety and Reliability Conference. Valencia, 